\title{
Magnetohydrodynamic Turbulence and the Geodynamo
}

\section{John V. Shebalin}

The ARES Directorate at JSC has researched the physical processes that create planetary magnetic fields through dynamo action since 2007. The "dynamo problem" has existed since 1600, when William Gilbert, physician to Queen Elizabeth I, recognized that the Earth was a giant magnet. In 1919, Joseph Larmor proposed that solar (and by implication, planetary) magnetism was due to magnetohydrodynamics (MHD), but full acceptance did not occur until Glatzmaier and Roberts solved the MHD equations numerically and simulated a geomagnetic reversal in 1995. JSC research produced a unique theoretical model in 2012 that provided a novel explanation of these physical observations and computational results as an essential manifestation of broken ergodicity in MHD turbulence. Research is ongoing, and future work is aimed at understanding quantitative details of magnetic dipole alignment in the Earth as well as in Mercury, Jupiter and its moon Ganymede, Saturn, Uranus, Neptune, and the Sun and other stars. Current computational research results showing effective dipole angle $\theta_{D}$ with respect to normalized rotation vector $\boldsymbol{\Omega}_{\mathrm{o}}$ are given in figure 1. (The dotted line is what would be expected if $\boldsymbol{\Omega}_{0}=\mathbf{0}$. Also, note that saturation occurs.)

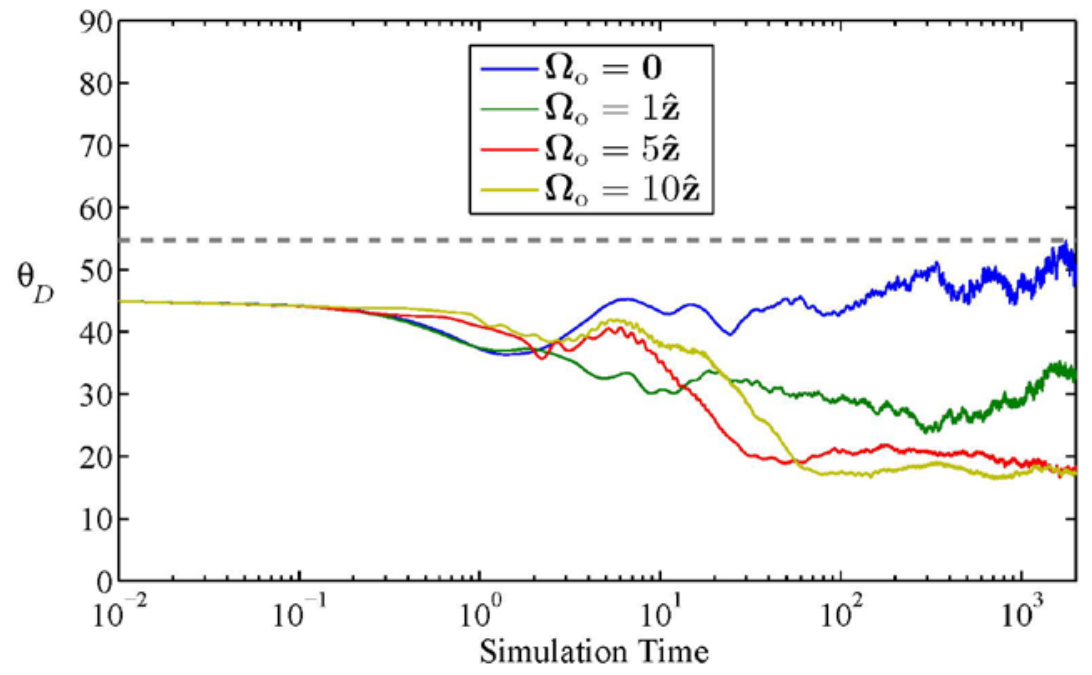

Figure 1.- Dipole angle with respect to rotation rate in a model geodynamo.

Current work focuses on the Earth because it is the planet observed most closely and the planet for which the best data exist. The geomagnetic field is of utmost importance to the growth and survival of life on Earth because it serves as a "magnetic bumper" that protects us from the solar wind, coronal mass ejection, and cosmic rays. The geomagnetic field is a primarily dipole field that allows for the existence of a stable atmosphere, without which Earth would probably look like Mars: dry, barren, and lifeless. However, the geomagnetic dipole field is not static, arising as it does from deep MHD flows, but changes over time - it has weakened about 10 percent since 1850, and its direction wanders (see figure 2) or even reverses (on average every 100,000 years). The geomagnetic field is intimately connected to the existence and location of the radiation belts, and its multipole 
components cause such features as the South Atlantic Anomaly, which adversely affects low-Earthorbit spacecraft, such as the International Space Station. A detailed knowledge of the geomagnetic field and how it changes in the short and long term is very important for understanding and predicting changes in the atmosphere (weather and climate) and for planning future near-Earth missions. Similar effects and concerns will occur in space missions that explore other planets in the solar system.

Understanding planetary magnetism is clearly important, and JSC research indicates that MHD turbulence plays a critical role. MHD processes underlie planetary magnetism and appear in the interior and exhaust plumes of plasma rocket engines, so that gaining an understanding in one area informs our efforts in the other. There is much that is still unknown, and ongoing research is expected to lead to important knowledge that can be applied to the planning and operation of future space missions and to a greater and more fundamental understanding of the origins, evolution, effects, and interactions of global magnetic fields generated within the Earth, other planets, and the Sun.

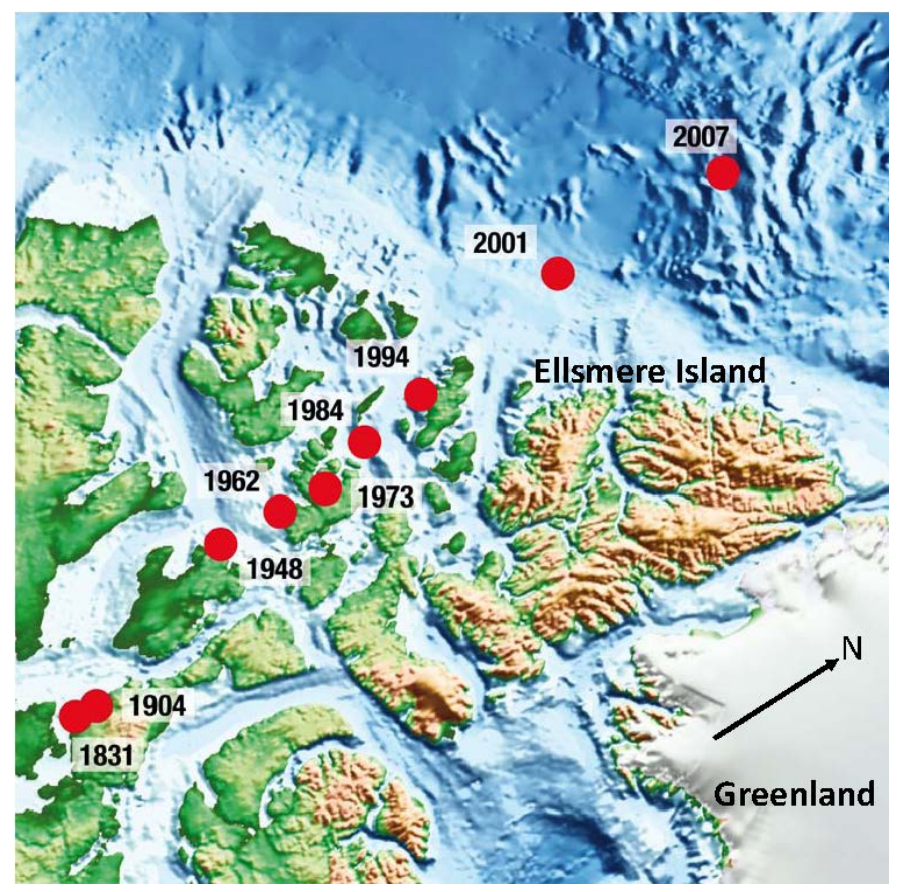

Figure 2.- Observed north dip poles from 1831-2007. (Image: Arnaud Chulliat, Institut de Physique du Globe de Paris). 